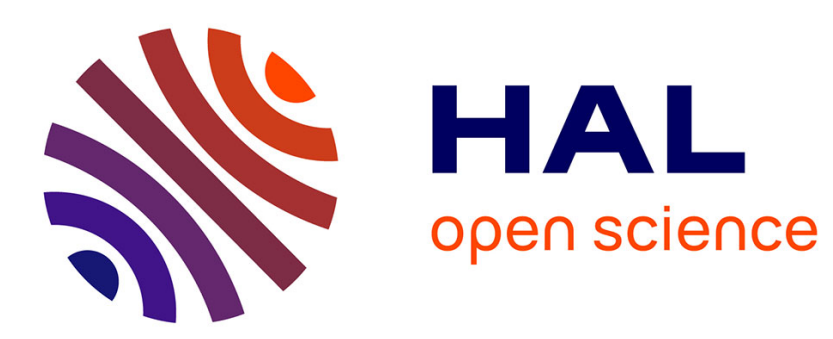

\title{
MULTIPLE CAPTURE AND IONISATION IN HIGH ENERGY ION-ATOM COLLISIONS
}

\author{
R. Gayet
}

\section{To cite this version:}

R. Gayet. MULTIPLE CAPTURE AND IONISATION IN HIGH ENERGY ION-ATOM COLLISIONS. Journal de Physique Colloques, 1989, 50 (C1), pp.C1-53-C1-70. 10.1051/jphyscol:1989106 . jpa-00229297

\section{HAL Id: jpa-00229297 https://hal.science/jpa-00229297}

Submitted on 1 Jan 1989

HAL is a multi-disciplinary open access archive for the deposit and dissemination of scientific research documents, whether they are published or not. The documents may come from teaching and research institutions in France or abroad, or from public or private research centers.
L'archive ouverte pluridisciplinaire HAL, est destinée au dépôt et à la diffusion de documents scientifiques de niveau recherche, publiés ou non, émanant des établissements d'enseignement et de recherche français ou étrangers, des laboratoires publics ou privés. 
JOURNAL DE PHYSIQUE

Colloque $\mathrm{C} 1$, supplément au $\mathrm{n}^{\circ} 1$, Tome 50 , janvier 1989

MULTIPLE CAPTURE AND IONISATION IN HIGH ENERGY ION-ATOM COLLISIONS

\author{
R. GAYET \\ Laboratoire des Collisions Atomiques, CNRS ER-260 Université \\ Bordeaux I, F-33405 Talence Cedex, France
}

Résumé - Les processus multiélectroniques qui interviennent dans les collisions ion-atome à haute énergie, sont examinés à la lumière de Modèles à Electrons Indépendants dont certaines limites apparaissent pour les processus de double capture. On montre qu'un tel modèle permet d'exhiber des comportements non perturbatifs du processus d'ionisation. C'est aussi un outil remarquable pour prédire la production d'ions de recul très chargés ainsi que leur distribution en énergie quand la vitesse d'impact du faisceau primaire est suffisamment élevée.

Abstract - Multiple electron processes occuring in high energy ion-atom collisions are investigated through Independent Electron Models (IEM), some limits of which are indicated for double capture processes. It is shown that an IEM permits to exhibit non perturbative behaviours of the ionisation process. It appears to be a powerful tool to predict multiply charged recoil ion production and recoil energy distribution when the impact velocity of the primary beam is high enough.

\title{
1 - INTRODUCTION
}

The present paper is a non-exhaustive overview of some multielectronic processes which occur in ion-atom collisions at high (though non relativistic) impact velocities. An atomic collision at high energy takes place when the initial and final orbital velocities of active electrons are smaller than the impact velocity $v / 1 /$. A useful criterion reads :

$$
E(\mathrm{keV} / \text { nucleon })>50 \sup \left\{\left|\epsilon_{\mathbf{i}}\right|,\left|\epsilon_{f}\right|\right\}
$$

where $\epsilon_{i}$ and $\epsilon_{f}$ are the orbital energies (in atomic units) of an electron which undergoes a transition from the orbital i to the orbital $f$ respectively (active electron). In practice, $\epsilon_{i}$ and $\epsilon_{f}$ could be orbital Hartree-Fock energies or similar energies. The present theoretical analysis is restricted to collisions satisfying condition (1). In such a situation, use of the Impact Paramater Treatment (IPT) is perfectly justified to get the electronic transition amplitude since the wavelength associated with the motion of the projectile is much smaller than the atomic dimensions.

Systematic experimental studies of multiple electron processes in high energy ion-atom collisions were made in the early 1970's /2,3,4/. The processes were found to be non negligible compared to single electron processes. For instance, Macdonald et al found a ratio of double to single capture by $\mathrm{F}^{9+}$ to amount to 0.2 in nitrogen $/ 2 /$ and to as much as 0.24 in argon /4/ for ions impinging at $25 \mathrm{MeV}$ and $17 \mathrm{MeV}$ respectively. Since then, much attention has been paid to atomic collisions where multielectronic processes are involved, i.e, simultaneous capture, excitation and/or ionisation.

In the present paper it is shown that, at high enough velocities, most theoretical studies can be made in the framework of the Independent Electron Model (IEM). In ref. $/ 2 /$, the authors already noticed : "Theoretical analysis $/ 5 / \ldots$ suggests that the ratio of double to single capture cross sections is a meaningful quantity... related to a probability for capture in a restricted domain of impact parameter". In fact, the IEM was established in a suitable form by McGuire and Weaver $/ 6 /$. The basic assumptions of the IEM and a brief sketch of it are recalled in section 2 . In the following sections, theoretical investigations of a few multielectronic processes are presented. Attempts are made to point out some striking features for each of them.

Section 3 deals with double capture from atoms by swift ions. The independent electron capture probability $P_{c}(\rho)$ as a function of the impact parameter $\rho$ is evaluated by means of the Continuum Distorted Wave Approximation (CDWA) which has been proved to be very reliable at high impact velocities $/ 7,8$. Iimitations of the IEM are shown for double charge transfer 
from $\mathrm{He}$ to $\mathrm{He}^{++}$. In this case, correlation effects are likely to play an important role in the double electron transfer $/ 9 /$.

In section 4, simultaneous capture and ionisation processes, for which experimental data have been obtained recently $/ 10,11,12 /$, are investigated within the IEM. It is shown that these processes may reveal hidden features of the independent electron probabilities. Using CDWA capture probabilities, Gayet and Salin /13/ showed that saturation effects on the ionisation probability $P_{i}(P)$ can be exhibited at small impact parameters, although these effects are not so visible in single ionisation processes where significant contributions are coming from large impact parameters. By saturation, one means that, at a given impact velocity $v$, the single ionisation probability $P_{i}(\rho)$ increases more slowly with the projectile charge $Z_{p}$ than the $Z_{p}^{2}$ behaviour predicted by the Born approximation. This non perturbative behaviour has been already predicted and confirmed in the case of single electron excitation where total cross sections are shown to reach finite limits for large values of $z_{p} / 14,15 /$. A recent work by Salin /16/ on single and double ionisation of helium by multicharged ions is mentioned since it is closely related to the topic of section 4.

In section 5, it is shown how joint use of the IEM and of the first Born approximation (BA1) permits to evaluate multiple ionisation of atoms by fast highly charged ions. Predictions of very slow multicharged recoil ion production are reported for typical primary ions beams of the GANIL ${ }^{*}$ (Caen, France) /17/. It is shown that their energy is enough to use the BAl, as well as the IEM, in order to obtain the probabilities $P_{i}(\rho)$ to eject one electron from a given subshell $i$. In addition, the contribution of capture to multiple ionisation is shown to be negligible, a situation which does not occur at intermediate or low impact energies $/ 18 /$. Furthermore, the energy distributions for recoil ions of a given charge state are obtained as by-products of the multiple ionisation probabilities.

Atomic units are used throughout unless otherwise stated.

\section{2 - THE INDEPENDENT ELECTRON MODEL}

Although the present sketch is inspired from papers of McGuire et al /6,19/, various levels of approximation should be distinguished. At the first stage, only transition amplitudes are considered. Hence a unique assumption is needed :

(a1) The total scattering wave functions $\Psi^{ \pm}$may be approximated by a product of single particle wave functions.

In order to see the consequences of assumption (al), let us have a look at a 2 electron problem in the impact parameter treatment (IPT) where the projectile nucleus follows a classical trajectory at a quasi-constant velocity $\mathbf{v}$. The internuclear distance $\mathbf{R}$ is written as :

$$
\overrightarrow{\mathrm{R}}=\overrightarrow{\mathrm{p}}+\overrightarrow{\mathrm{v} t}=\vec{\rho}+\overrightarrow{\mathrm{z}} \quad ; \quad \overrightarrow{\mathrm{v}} \cdot \vec{\rho}=0
$$

where $\vec{\rho}$ is the impact parameter and $t$ the time $(t=0$ when $\vec{R}=\vec{\rho})$. Let $\Phi_{i}\left(\overrightarrow{r_{1}}, \overrightarrow{r_{2}}, t\right)$ and $\Phi_{f}\left(\overrightarrow{r_{1}}, \vec{r}_{2}, t\right)$ be the asymptotic initial and final states of the system respectively. The vector $\widehat{r_{k}}(k=1,2)$ locates electron $k$ in a given frame.

Let $\Psi^{ \pm}\left(\overrightarrow{r_{1}}, \overrightarrow{r_{2}}, t\right)$ be the scattering wave functions such that :

$$
\Psi_{i}^{+} \underset{t \rightarrow-\infty}{\longrightarrow} \Phi_{i} \quad ; \quad \Psi_{f} \underset{t \rightarrow+\infty}{\longrightarrow} \Phi_{f}
$$

The transition amplitude from $i$ to $f$ reads :

$$
A_{i f}=\left\langle\Psi_{f} \mid \Psi_{i}^{+}\right\rangle
$$

$\Psi^{ \pm}$are solutions of the equation :

$$
\left(\mathscr{H}-\mathbf{i} \frac{\partial}{\partial t}\right) \Psi^{ \pm}=0
$$

where the full hamiltonian $\mathcal{H}$ is :

$$
\mathscr{H}=\mathrm{H}_{\mathbf{i}}+\mathrm{V}_{i}=\mathrm{H}_{\mathbf{f}}+\mathrm{V}_{f}
$$

\footnotetext{
"GANIL is "Grand Accélérateur National d'Ions Lourds"
} 
In equation (6), $\mathrm{H}_{j}$ and $\mathrm{H}_{f}$ are the hamiltonians of the asymptotic channels $i$ and $f$ respectively. $v_{i}$ and $v_{f}$ are the corresponding target-projectile interactions. Thus one has :

$$
\left(\mathrm{H}_{i}-i \frac{\partial}{\partial t}\right) \varphi_{i}=\left(\mathrm{H}_{f}-i \frac{\partial}{\partial t}\right) \varphi_{f}=0
$$

In the entrance channel i, assumption (al) means :

$$
\Psi\left(\overrightarrow{r_{1}}, \overrightarrow{r_{2}}, t\right) \simeq \psi_{i_{1}}^{+}\left(\overrightarrow{r_{1}}, t\right) \psi_{i_{2}}^{+}\left(\overrightarrow{r_{2}}, t\right)
$$

It is possible only if $\mathcal{H}$ may be approximated by a separable hamiltonian, i.e. :

$$
\mathcal{H} \simeq \mathrm{h}_{1}\left(\overrightarrow{\mathrm{r}_{1}}, \overrightarrow{\mathrm{R}}\right)+\mathrm{h}_{2}\left(\overrightarrow{\mathrm{r}_{2}}, \overrightarrow{\mathrm{R}}\right)
$$

with :

$$
\left(h_{1}-i \frac{\partial}{\partial t}\right) \psi_{i_{1}}^{+}\left(\vec{r}_{1}, t\right)=\left(h_{2}-i \frac{\partial}{\partial t}\right) \psi_{i_{2}}^{+}\left(\overrightarrow{r_{2}}, t\right)=0
$$

Equations (6) and (9a,b) implie that $H_{i}$ is also separable since $v_{i}$ is zero at large internuclear distances. Therefore, $\Phi_{i}$ may also be written as a product of single electron wave functions. Of course, similar conclusions are drawn for the exit channel $f$. Thus, the transition amplitude (4) becomes

$$
A_{i f} \simeq\left\langle\psi_{f_{1}}^{-} \mid \psi_{i_{1}}^{*}\right\rangle\left\langle\psi_{f_{2}}^{*} \mid \psi_{i_{2}}^{+}\right\rangle=A_{i_{1} f_{1}} A_{i_{2} f_{2}}
$$

Finally, the first stage of the IEM permits us to define the transition amplitude as a product of single electron transition amplitudes. From equation ( $9 \mathrm{a}$ ), it is obvious that the assumpition (al) means that two-electron potentials such as $\left|\overrightarrow{r_{1}}-\overrightarrow{r_{2}}\right|^{-i}$ can be approximated by suitable one-electron interaction terms. Hence, if the projectile is a non-bare ion, it should be considered as a point charge.

This first stage may be useful for a pure two electron system in a singlet state. However, it is difficult to cope with it when more than 2 electrons are involved.

Herce, a second stage of approximation is often introduced for complex multielectronic processes at high impact velocities. It deals with one electron transition probabilities. The following assumptions are necessary :

(a2) All the interactions experienced by active electrons may be approximated by single-electron potentials.

(b2) One active electron experiences the same approximate single-electron potentialsin the entrance and exit channels as well as diring the transition.

(c2) The probability for a given multielectronic transition to occur is the result of a combinative calculus with the probabilities for each electron either to stay on its initial orbital or to occupy another free one.

Assumption (a2) means that correlation effects are neglected, i.e., two electron potentials are approximated by single electron ones. The assumption (b2) permits an easy calculation of the probability for each electron to undergo a change. While an electron moves out its initial orbital, the other ones are considered to the "frozen" in their initial orbital. It appears to be very useful to evaluate the probability to eject one electron from a given orbital whatever occurs to the other ones. Finally, the combination of probabilities in using (c2) should take into account the exclusion Pauli principle. Hence, when, one electron occupies a final orbital, a second one with the same spin cannot occupy it. Practically, this constraint may be ignored when dealing with simultaneous ejection of several electrons into the continuum. However, it must be considered seriously for multiple electron capture processes.

Obviously no interference effects can be account for in this second stage hereafter referred to as IEM2 while the first one is referred to as IEM1.

\section{3 - DOUBLE ELECTRON CAPTURE BY SWIFT IONS}

Theoretical investigations for multiple charge transfer appeared in the late 1970's. Lin /20/ studied double $\mathrm{K}$-shell to K-shell electron transfer in the framework of IEMI. He considered the process as a two step mechanism, each electron being captured separately. The interelectronic coulomb interaction $r_{12}^{-1}$ is introduced in electron-nucleus interactions by means of a screening charge. The interactions with outer-shel1 electrons, when existing, are 
neglected. First order calculations, like OBK calculations $/ 21,22 /$ were already known to be inadequate for single charge transfer $/ 7$ and ref. therein/ as well as double one /23/. Therefore, he used a three-state two-centre atomic expansion to solve equation (5) with $\mathscr{H}$ approximated as in equation (9a). Double capture by $\mathrm{H}^{+}$in He provides an instance of what the 3 states are :

$\mathrm{H}^{+}-\mathrm{He}\left(1 \mathrm{~s}^{2}\right), \mathrm{H}(1 \mathrm{~s})-\mathrm{He}^{+}(1 \mathrm{~s})$ and $\mathrm{H}^{-}\left(1 \mathrm{~s}^{2}\right)-\mathrm{He}^{++}$.

Lin applied his model in the intermediate impact velocity region where similar methods made reasonable predictions. The agreement is good neither for $\mathrm{H}^{+}$- He collision (fig. 1), nor for double $K$ to $K$ capture by $\mathrm{O}^{8+}$ and $\mathrm{N}^{7+}$ in neon (fig. 2) while it is acceptable for $\mathrm{He}^{++}$on $\mathrm{He}$ and excellent for double capture by $\mathrm{F}^{9+}$ in neon (fig. 2). Also displayed on fig. 1 is the calculation of Roy et al /24/ who solved the impact parameter Schrödinger equation (5) with the full exact hamiltonian. A three-state two centre atomic expansion was also used but, in contrast to Lin's model, correlated wave functions for $\mathrm{H}^{-}$and $\mathrm{He}$ as well as antisymetrisation for both electrons in the intermediate state is properly accounted for in the expansion. Their only approximation, except the truncation of the basis set, is that the two-centre excharige integrals are evaluated approximately. Except at lower energies, their results disagree with experimental data $/ 25,26 /$ more than those of Lin. As to first Born like calculations made by Gerasimenko /27/, they appear to be two ordersof magnitude above experimental data.For all theories, the higher the energy, the worse the agreement with experiment. Then one expects limited atomic expansions to be not working at high energy. Indeed, the comparison of Lin's results with experimental data is not very significant for double capture by $0^{8+}$ or $\mathrm{N}^{7+}$ ions since only one experimental point is known. Then, one could think, following Lin, that his model is expected to work reasonably well at intermediate impact velocities for $z_{p}, z_{T} \gg 1$ where $z_{p}$ and $z_{T}$ are the charges of the projectile and of

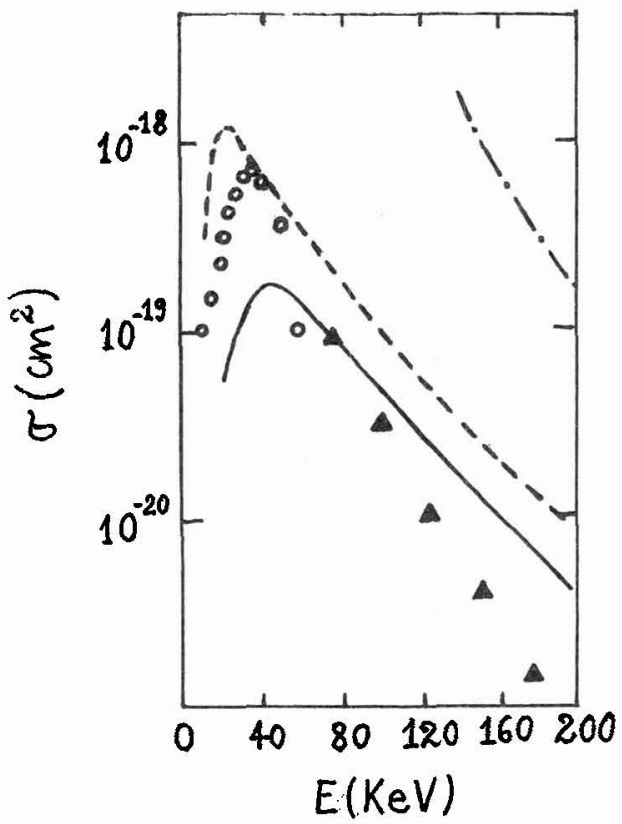

Fig. 1 - Total cross sections $\sigma$ for double capture by protons in helium. Experimental ; 0 ref. $/ 25 / ;$ ref. $/ 26 /$. Theoretical :

- Lin $/ 20 /$; -.- Roy et al $/ 24 /$; . - Gerasimenko $/ 27 /$.

the target respectively. Indeed, the approximation of replacing $r_{12}^{-1}$ by screening charges and the representation of two-electron wave functions by product of hydrogenic ones are expected to be more valid with increasing $Z_{p}$ and $Z_{T}$.

However, at high energy, three state as well as two state atomic expansions converge to the OBK approximation. Gayet et al /7/ showed that the failure of the latter one lies in the incorrect treatment of the Coulomb asymptotic conditions. When the right conditions are introduced in the calculations of a first Born matrix element, acceptable predictions for single electron capture are obtained /28/. However, the capture amplitude can no longer be given in an analyticalform. In fact, at not too high impact velocities, it provides results 
comparable to the ones of the Continuum Distorted Wave Approximation (CDWA). The latter theory is an outcome of a perturbation treatment of a 3 body scattering theory which appears very useful when one particle (the electron) is much less massive than both other ones. Introducing the exact asymptotic conditionsdue to the Coulomb potentials, which occur with three charged particles, provides the CDWA amplitude to first order of perturbation /29/. For single electron capture, CDWA amplitudes can be given analytical forms even for complex atoms or ions $/ 7 /$. It is demonstrated to be very reliable at impact energies given by equation (1) where the factor 50 is replaced by 80 . Although a generalisation of the theory to more than 1 electron is easy, it can be shown that no analytical form can be given to the CDWA amplitude for multiple capture $/ 30 /$. Therefore, the first theoretical investigation of double capture with CDWA has been made in the framework of IEMI by Gayet et a1 /9/. A brief sketch of their: derivation is given hereafter. It is made in the IPT in a way analogous to the first CDWA derivation made by Cheshire /31/.

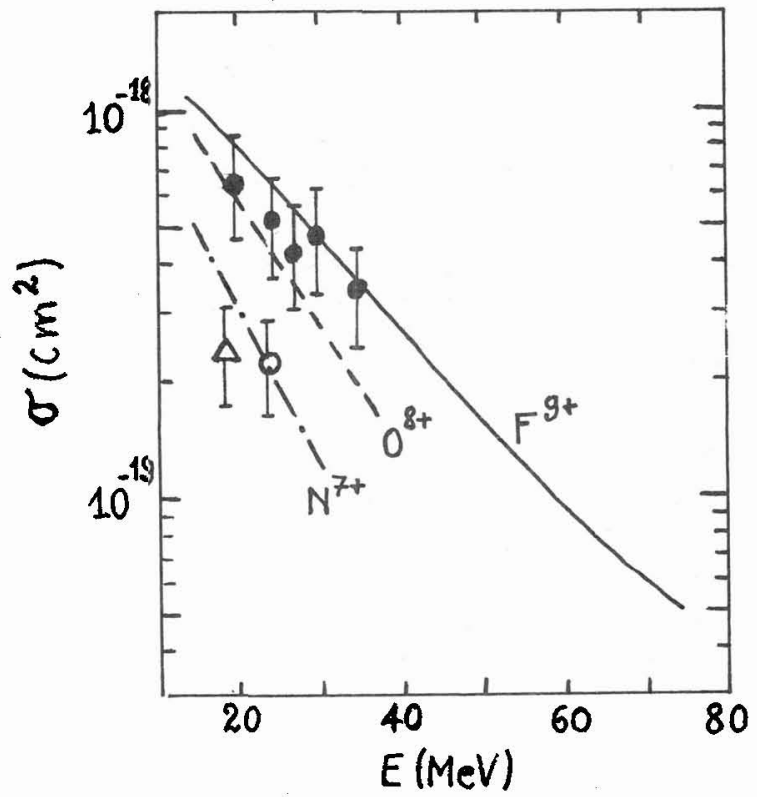

Fig. 2 - Total cross section $\sigma$ for double capture from the K-shell of neon by bare nuclei as a function of the lab-impact energy. Experimental data $/ 23 /: \bullet \mathrm{F}^{9+}+\mathrm{Ne} ; 00^{8+}+\mathrm{Ne} ; \Delta \mathrm{N}^{7+}+$ Ne. Theoretical results of $\operatorname{Lin} / 20 /:-F^{9+}, \cdots 0^{8+}, \cdots, \cdot \mathrm{N}^{7+}$

Consider a collision between a bare ion and a helium-like target. Let P, T, 1 and 2 be the projectile, the target nucleus and the electrons respectively. Let $\mathrm{z}_{\mathrm{p}}$ and $\mathrm{z}_{\mathrm{T}}$ be the charges of $\mathrm{P}$ and $\mathrm{T}$. The electronic Hamiltonian is (see fig. 3)

$$
\mathrm{H}=-\frac{\nabla_{1}^{2}}{2}-\frac{\nabla_{2}^{2}}{2}-\frac{\mathrm{z}_{\mathrm{p}}}{\mathrm{s}_{1}}-\frac{\mathrm{Z}_{\mathrm{p}}}{\mathrm{s}_{2}}-\frac{\mathrm{Z}_{\mathrm{T}}}{\mathrm{x}_{1}}-\frac{\mathrm{Z}_{\mathrm{T}}}{\mathrm{x}_{2}}+\frac{1}{\mathrm{r}_{12}}
$$

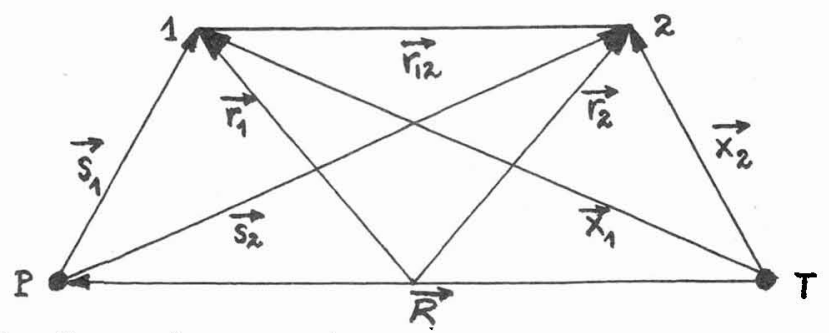

fig. 3 - nuclear and electronic coordinates (see text)

The initial and final channel hamiltonians and perturbing potentials are respectively 


$$
\begin{aligned}
& \mathrm{H}_{\mathrm{i}}=-\frac{\nabla_{1}^{2}}{2}-\frac{\nabla_{2}^{2}}{2}-\frac{\mathrm{z}_{\mathrm{T}}}{\mathrm{x}_{1}}-\frac{\mathrm{z}_{\mathrm{T}}}{\mathrm{x}_{2}}+\frac{1}{\mathrm{r}_{12}} \\
& \mathrm{H}_{\mathrm{f}}=-\frac{\nabla_{1}^{2}}{2}-\frac{\nabla_{2}^{2}}{2}-\frac{\mathrm{z}_{\mathrm{p}}}{\mathrm{s}_{1}}-\frac{\mathrm{z}_{\mathrm{p}}}{\mathrm{s}_{2}}+\frac{1}{\mathrm{r}_{12}} \\
& \mathrm{v}_{\mathrm{i}}=-\frac{\mathrm{z}_{\mathrm{p}}}{\mathrm{s}_{1}}-\frac{\mathrm{z}_{\mathrm{p}}}{\mathrm{s}_{2}} \\
& \mathrm{v}_{\mathrm{f}}=-\frac{\mathrm{z}_{\mathrm{T}}}{\mathrm{x}_{1}}-\frac{\mathrm{z}_{\mathrm{T}}}{\mathrm{x}_{2}}
\end{aligned}
$$

The eigenfunction associated with $\mathrm{H}_{i}$ is /7/:

$$
\Phi_{i}=\varphi_{i}\left(\overrightarrow{r_{1}}, \overrightarrow{r_{2}}\right) \exp \left[-\frac{1}{4} i v^{2} t-i \epsilon_{i} t-\frac{1}{2} \mathbf{1} \vec{v}\left(\overrightarrow{r_{1}}+\overrightarrow{r_{2}}\right)\right]
$$

The vectors $\overrightarrow{r_{1}}$ and $\overrightarrow{r_{2}}$ are referred to the midpoint of PT.

In expression (14); $\varphi_{i}$ and $\epsilon_{i}$ are respectively eigenfuctions and eigenenergies of $H_{i}$, $\vec{\nabla}$ is the relelative velocity between the nuclei. The time dependent Schrödinger equation of the impact parameter approximation

$$
\left.\left(H-i \frac{d}{d t}\right)_{\overrightarrow{r_{1}}, \vec{r}_{2}}\right) \Psi_{i}\left(\overrightarrow{r_{1}}, \overrightarrow{r_{2}}, t\right)=0
$$

has to be solved with the condition

$$
\Psi_{i} \underset{t \rightarrow-\infty}{\longrightarrow} \Phi_{i} \exp \left[-2 i v^{-1} z_{p} \ln \left(v R-v^{2} t\right)\right]
$$

Let us write $\Psi_{i}$ as

$$
\Psi_{i}=\Omega_{i}\left(\overrightarrow{r_{1}}, \overrightarrow{r_{2}}\right) \Phi_{i}
$$

which gives the equation for $\mathfrak{L}_{i}$

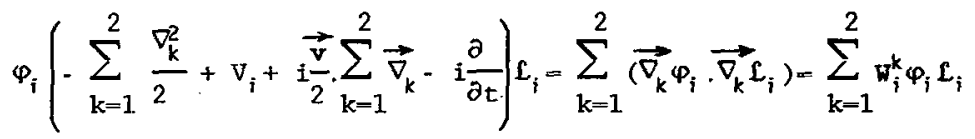

The CDW approximation consists of neglecting the RHS of (18) $/ 31 /$. Then the solution is a product of independently distorted wavefunctions for each electron whether or not the independent electron model is used.

$$
\mathfrak{L}_{i}^{C D W}=\prod_{k=1}^{2} \mathcal{L}_{i}^{k}\left(\overrightarrow{s_{k}}\right)
$$

Then, the solution of (18), with the exact asymptotic conditions imposed by $v_{i}$ in equation (13a) is /32/:

$\mathcal{L}_{i}^{k}=\exp \left(\frac{1}{2} \pi \frac{Z_{p}}{v}\right) \Gamma\left(1-i \frac{Z_{p}}{v}\right){ }_{1} F_{1}\left(i \frac{Z_{p}}{v} ; 1 ; i v s_{k}+i \vec{v} \cdot \overrightarrow{s_{k}}\right)$

A similar derivation can be made with the exit channel to obtain $\Psi_{f}$ as

$$
\Psi_{f}=\mathfrak{L}_{f}^{1}\left(\overrightarrow{x_{1}}\right) \mathfrak{L}_{f}^{2}\left(\overrightarrow{x_{2}}\right) \Phi_{f}
$$


The electron-electron interaction is included in the channel Hamiltonians. We introduce now the independent electron approximation which is required to make the integration in the $T-$ matrix feasible. According to $(9 a)$, we approximate $\mathrm{H}_{j}$ as

$$
H_{i}=\sum_{k=1}^{2} H_{i}^{k}\left(\overrightarrow{x_{k}}\right)
$$

where

$$
H_{i}^{k}=-\frac{\nabla_{k}^{2}}{2}-\frac{Z_{T}}{x_{k}}+v_{i}^{k}\left(\overrightarrow{x_{k}}\right)
$$

In expression (23), $v_{i}^{k}\left(\vec{x}_{k}\right)$ is an average potential which takes into account the interaction of the electron $k$ with the other one. For the entrance channel we can use the Hartree-Fock potential so that an eigenfunction of $\mathrm{H}_{\dot{j}}^{k}$ is the initial $\mathrm{HF}$ orbital of electron $k$. There is some ambiguity in the similar choice of $v_{f}^{k}$ for the final channel Hamiltonian. The use of Hartree-Fock potential implies that the relaxation time for the final state of electron $k$ under its interaction with the other captured electron is much shorter than the collision time. This is not necessarily the case at high energies. We consider therefore two extreme situations :

(i) $v_{f}^{k}=0$. No relaxation of the final state of electron $k$ occurs during the collision time, so that its final orbital is a hydrogenic one in the field of the nucleus $z_{p}$. In other words, the electron-electron interaction is switched off during the collision.

(ii) The final orbital is the Hartree-Fock orbital for the helium-like state formed around the projectile. In other words, the two electrons are captured as a pair of strongly interacting particles (although this interaction is treated as an average in the sense of the HF-model - which is a necessary condition to get an independent-electron model).

In these two cases, and considering only singlet states, both eigenfunctions associated with the entrance and exit hamiltonians may be written as simple products of two one-electron wave functions i.e.:

$$
\varphi_{i}=\prod_{k=1}^{2} \varphi_{i}^{k}\left(\overrightarrow{x_{k}}\right) ; \varphi_{f}=\prod_{k=1}^{2} \varphi_{f}^{k}\left(\overrightarrow{s_{k}}\right)
$$

The discussion above shows that in our theory the electron-electron repulsion enters the problem only through the final and initial bound wavefunctions $\varphi_{j}(j=i, f)$. We can therefore predict that our approximation improves as the role of electron correlation effects in $\varphi_{j}$ decreases (e.g. by increasing nuclear charges of target or projectile).

The treatment is now very close to that of McGuire and Weaver $/ 6 /$. One defines the channel distorted wavefunctions

$$
x_{j}=\prod_{k=1}^{2} x_{j}^{k}=\prod_{k=1}^{2} \varphi_{j}^{k} \mathfrak{L}_{j}^{k} \quad(j=i, f)
$$

where $x_{j}^{k}$ is the solution of the equation

$$
\left(H_{j}^{k}+u_{j}^{k}-i \frac{d}{d t}\right) x_{j}^{k}=0
$$

In (26) $\mathrm{U}_{\mathbf{j}}^{\mathbf{k}}$ is the usual distortion potential of the CDW approximation for electron $k$ in channel $\mathrm{j} / 7 /$. When the two-electron scattering wavefunction of eq. (21) may be approximated in the form

$$
\Psi_{f}=\prod_{k=1}^{2} \psi_{f}^{k}
$$

the transition amplitude is

$$
A_{i f}=\lim _{t \rightarrow-\infty}\left\langle\Psi_{f} \mid x_{i}\right\rangle=\prod_{k=1}^{2} \lim _{t \rightarrow-\infty}\left\langle\psi_{f}^{k} \mid x_{i}^{k}\right\rangle=\prod_{k=1}^{2} a_{i f}^{k}
$$


where

$$
a_{i f}^{k}=\lim _{t \rightarrow-\infty}\left\langle\psi_{f}^{k} \mid x_{i}^{k}\right\rangle=-i \int_{-\infty}^{+\infty} d t\left\langle\psi_{f}^{k}\left|w_{i}^{k}\right| x_{i}^{k}\right\rangle
$$

$a_{i f}^{k}$ is the one-electron transition amplitude for electron $k$. Replacing $\psi_{f}^{k}$ by $\chi_{f}^{k}$ in the last term of (29), one obtains the CDWA amplitude for one-electron capture. If the orbitals $\varphi_{f}^{k}$ can be expanded on a finite set of slater-type orbitals, then the codes of Belkic et al /33/ may be used to evaluate $a_{i f}^{k}$ after some modifications indicated in $/ 9 /$.

The present theory has been used to investigate two reactions for which experiments are available :

$$
\begin{aligned}
& \mathrm{He} e^{2+}+\mathrm{He}\left(1 s^{2}\right) \rightarrow \mathrm{He}\left(1 s^{2}\right)+\mathrm{He}^{2+} \\
& \mathrm{F}^{9+}+\mathrm{Ar} \rightarrow \mathrm{F}^{7+}\left(1 \mathrm{~s}^{2}\right)+\mathrm{Ar}^{2+}
\end{aligned}
$$

In the case of reaction (31), only capture from the $\mathrm{L}$ shell of a argon has been considered since it dominates the capture process at the energies involved in the experiment of Ferguson et al $14 \%$. In order to test our model, two types of calculations have been performed

corresponding to the above approximations (i) and (ii), that is the $1 s^{2}$ final state is either a product of hydrogenic orbitals with charge $z_{p}$ for case (i) or, a product of HF orbitals (as given by Clementi and Roetti /34/) for case (ii).

In both cases, the initial orbitals are the Hartree-Fock orbitals of clementi and Roetti, an assumption which is realistic since we know that in the experiments, the targets are prepared In their ground state. Note that the hypothesis that the orbitals of the passive electrons are frozen means that the $\mathrm{Ar}^{2+}$ ion is left in a state which is not an eigenstate of its Hamiltonian, an assumption which has already been shown to be excellent for one electron capture $/ 7 /$.

In the comparison between theory and experiment, one should note that the experiments give double capture cross sections summed over all final states whereas the theoretical. cross sections are for (30) and (31).

Results for reaction (30) are given in table 1. Although fairly good agreement with experiment is found in case (i) in the energy range where CDWA works well for one-electron capture, no definitive conclusion can be drawn since the discrepancy between the calculations (i) and (ii) in slope and magnitude reveals that two-electron capture is very sensitive to the choice of final orbitals. The calculations of Theisen and McGuire /35/are in good agreement with the experimental data in the intermediate-energy region. Three-state two-centre results of Lin, reported by Theisen and McGuire /35/, appears in even better agreement in this region but seem to exhibit a wrong behaviour at high energy ; a similar feature shows up in Theisen and McGuire's calculations.

Table 1. - Total electron capture cross section by $\mathrm{He}^{2+}$ in helium. Theoretical results are only for capture into the ground state of helium : (i) and (ii) CDWA calculations (see text), TSAE : two-state atomic expansion of Theisen and McGuire/35/. Experimental results : capture into al.1 states of helium $/ 36 /$. The superscript is the power of ten to which the cross section is raised. Arrows indicate the region of validity of the CDW approximation for the one electron charge exchange process.

$\begin{array}{ccccc}\text { E(keV) } & (i) & (\text { ii }) & \text { TSAE } & \text { Experiment } \\ 500 & 1.6^{-17} & \sqrt{1.3^{-17}} & 3.2^{-18} & 5.1^{-18} \\ 750 & \sqrt{1.8^{-18}} & 1.1^{-18} & 7.0^{-19} & 9.5^{-19} \\ 1000 & 3.1^{-19} & 1.7^{-19} & 2.1^{-19} & 2.6^{-19} \\ 1400 & 3.4^{-20} & 1.7^{-20} & & 3.6^{-20}\end{array}$

Results for reaction (31) are given in table 2. The nuclear attraction felt by the two captured electrons in the entrance and exit channel of (31) dominates the electron-electron repulsion. Hence, one would expect the results to be less sensitive to the form of the final orbital. This is born out by our results since the discrepancy between the results (i) and (ii) is much smaller than in the case of reaction(30). Good agreement is obtained between both sets of results and experiment.

Table 2 - Total double electron capture cross section by $\mathrm{F}^{9+}$ in argon. Theoretical CDWA results are capture from the $L$ shell of argon into the ground state of $F^{7^{+}}$in cases (i) and (ii) (see text). Experimental data are from Ferguson et al $/ 4 /$. The superscript is the power of ten to which the cross section is raised. Arrows indicate the region of validity of the CDW approximation for the one-electron charge exchange process. 


$\begin{array}{cccc}\mathrm{E}(\mathrm{MeV}) & (i) & (\mathrm{i} i) & \text { Experiment } \\ 30 & 1.8^{-17} & 2.0^{-17} & 5.7^{-18} \\ 35 & 9.8^{-18} & 1.1^{-17} & 4.0^{-18} \\ 40 & 5.6^{-18} & 5.9^{-18} & 3.0^{-18} \\ 45 & 3.4^{-18} & 3.4^{-18} & 2.2^{-18} \\ 50 & \sqrt{2.1^{-18}} & \sqrt{2.1^{-18}} & 2.0^{-18} \\ 55 & 1.3^{-18} & \sqrt{1.3^{-18}} & 1.5^{-18} \\ 62 & 7.4^{-19} & 7.1^{-19} & \end{array}$

It has been shown how the CDW approximation can be used to study multiple electron capture at high energies. However some difficulties appear with the treatment of the interaction between the captured electrons. These correlation effects seem to be of great importance in the case of double electron capture from helium by alpha particles. Both the magnitude and slope of the total cross section versus energy are sensitive to these effects. A similar feature was already mentioned for double capture by protons in helium (see fig. 1). These difficulties seem related to the independent electron model itself. Thus, more experimental and theoretical work on this problem is still necessary to clarify the situation. Full correlated CDWA calculation are not out of reach. They would help to clarify the situation.

Anyway, joint application of CDWA and IEM for multiple electron capture by highly charged ions at high velocities appear to give reliable predictions.

\section{4 - SIMULTANEOUS CAPTURE AND IONISATION OF HELIUM}

Recent coincidence experiments /10,11,37/ have been performed to study the reactions :

$$
\begin{aligned}
\mathrm{Iq}^{+}+\mathrm{He}\left(I s^{2}\right) & \rightarrow \mathrm{I}(q-1)++\mathrm{He}^{+} \\
& \rightarrow \mathrm{I}(q-1)++\mathrm{He}^{++}+e^{-}
\end{aligned}
$$

where $\mathrm{I}^{\mathrm{q}^{*}}$ is an ion of charge $\mathrm{q}$. Since the helium target is in a singlet state, the application of the IEM2 is particularly simple. Let us denote by $P_{c}(P)$ and $P_{i}(P)$ the probabilities of capture and ionisation, respectively, for a single electron in a collision with impact parameter $P$. In the present case, to be consistent with the independent electron model, $P_{c, j}(\rho)$ has been calculated using au HF description of the initial He(ls ${ }^{2}$ ) state and assuming that the second electron is not affected while the first one is ejected or captured. The single capture cross sections without or with simultaneous ionisation are

$$
\begin{aligned}
& \sigma_{c}=2 \pi \int_{0}^{\infty} \rho \mathrm{d} \rho 2 \mathrm{P}_{c}(\rho)\left(1-P_{i}(\rho)\right) \\
& \sigma_{c i}=2 \pi \int_{0}^{\infty} \rho \mathrm{d} \rho 2 \mathrm{P}_{c}(\rho) \mathrm{P}_{\mathrm{i}}(\rho)
\end{aligned}
$$

respectively, if we neglect any other channel (e.g. double capture). The evaluation of $P_{c}(\rho)$ has been made using the CDWA codes of Belkit et al /33/ to calculate the amplitudes of single capture. Then the Hankel transforms of the latter ones permit to evaluate $P_{c}(\rho) / 38 /$.

Capture into excited states $n_{f}$, which plays an appreciable role in most cases considered here, is accounted for through the $n_{f}^{-3}$ law (see e.g. /7/) :

$P_{c}(\rho) \simeq \sum_{n_{f}=1}^{n_{0}} p_{c}\left(n_{f}, \rho\right)+p_{c}\left(n_{0}, \rho\right) n_{0}^{3} \sum_{n_{f}=n_{0}+1}^{\infty} n_{f}^{-3}$

where $p_{c}\left(n_{f}, \rho\right)$ is the probability of capture into the final state $n_{f}$. In any case, we have checked that $n_{0}$ is large enough to ensure an $n_{f}^{-3}$ behaviour of $p_{c}\left(n_{f}, \rho\right)$. For instance, a value of $n_{0}$ as large as ten is required for $0^{8+}$ projectiles. the accuracy in the calculations of $P_{c}\left(n_{f}, \rho\right)$ has been verified by an integration over $\rho$ in order to get the total capture cross sections. Then, the latter is compared with that given by the standard CDW2 code of Belkid et al $/ 33 /$. The accuracy is found to be better than $1 \%$ in each case. 
The ionisation probability has been calculated using two different approaches : the Born approximation and the Multipole Expansion Defined on One Centre (MEDOC), a procedure introduced by Cheshire and Sullivan $/ 42 /$.

In the first Born calculations, the initial (bound) and final (continuum) states of the ejected electron are known from a numerical integration of the Schrödinger equation for an electron in the field of a potential $V_{Y}(r)$, where $\vec{r}(r, \theta, \varphi)$ is the position vector of the electron with respect to the target nucleus; for convenience, use has been made of an analytical form of $\mathrm{V}_{\mathrm{T}}(\mathrm{r})$.

For low energies or large projectile charges $Z_{p}, P_{i}(p)$ as calculated with the Born approximation, may be larger than one at small impact parameters. Such saturation effects are wel1 known in the case of single excitation of a target by heavy-ion impact /14/. The perturbation approach is clearly unsuitable to this case since the Born series is equivalent to a series in powers of $z_{p}$. Therefore, we made use of the MEDOC non-perturbative numerical method, which consists in expanding the one-electron wavefunction in partial waves around the target :

$$
\Psi(\vec{x}, \mathrm{t})=\sum_{\ell} \mathrm{Y}_{\ell}^{m}(\theta, \varphi) \mathrm{L}_{\ell_{m}}(x, t)
$$

The time-dependent Schrödinger equation describing the motion of the electron in both the potential $V_{T}(r)$ and the time-dependent field of the projectile can be put in the form of a set of coupled differential equations in both $r$ and $t$ of the type :

$\left(-\frac{1}{2} \frac{d^{2}}{d x^{2}}+i \frac{d}{d r}+R_{\ell_{\mathfrak{m}}}^{\ell_{m}}(r, t)\right) L_{\ell_{m}}(r, t)=\sum_{\ell^{\prime} m^{\prime}} R_{\ell^{\prime} \ell_{m}}^{\ell_{m}}(r, t) L_{\ell^{\prime} m}(r, t)$

where $\mathrm{R}_{\ell, \mathrm{m}}^{\ell_{\mathrm{m}}}$, are matrix elements of the potential between spherical harmonics $\mathrm{Y}_{\mathrm{m}}^{\dot{\ell}}$ and $\mathrm{Y}_{\ell}^{m}$,. These equations are integrated by a finite difference procedure, in a code made by Salin (unpublished yet).

Two poüts should be stressed.

(i) The method cannot be expected to be adequate when the capture probability is large. This does not occur in the cases considered here.

(ii) The limitation, therefore, is on the number of partial waves included in the expansion (not on the strenght of the potential). In the present case we have included $\ell=0,1$. A discussion on the accuracy of this approximation is given below together with the analysis of the results.

The most important fact is illustrated in figure 4 : the capture probability decreases very rapidly for all the cases considered here when $\rho>0.5 \mathrm{au}$. This is in sharp contrast with the ionisation probability which decreases very slowly with impact parameter due to the dominant contribution of the dipole term in the ionisation amplitude $(\Delta \ell=1)$. To evaluate the total ionisation cross section in the same energy range, one must integrate up to impact parameters larger than 10 au. Furthermore, the monopole term $(\Delta \ell=0)$, which plays a weak role in the total cross section, is dominant at small impact parameters. Therefore, no conclusion on simultaneous capture and ionisation can be drawn by extrapolating qualitative ideas on total ionisation processes:

A second important fact is that the saturation effects mentioned above are much stronger for small impact parameters (figure 4). For $0^{8+}$ impact, the Born approximation to $P_{i}(\rho)$ gives probabilities larger than one at small impact parameters. Thus, deviations from the Born approximation are much more visible in simultaneous capture and ionisation processes than in single ionisation collisions. Hence, measurements on double active-electron processes in heavy ion-atom collisions at high impact velocities are very welcome.

Our results for $\sigma_{e j} / \sigma_{c}$ are compared with experimental data in figure 5 and in table 2 . Besides the use of the independent-electron model, their main limitation lies in the introduction of $\ell=0,1$ only in the MEDOC calculations. We can evaluate the accuracy of this approximation by looking at the convergence of the Born expansion with $\ell$. If we include only $\ell=0,1$ in the Born approximation, then the error in $P_{i}(P)$ is of the order of $10 \%$. Of

course we cannot completely trust this argument for $0^{8+}$ impact since the saturation effect is so strong that the Born results are unrealistic. However, even in this case, the contribution of $\ell$ values larger than one decreases as $\rho$ decreases. 


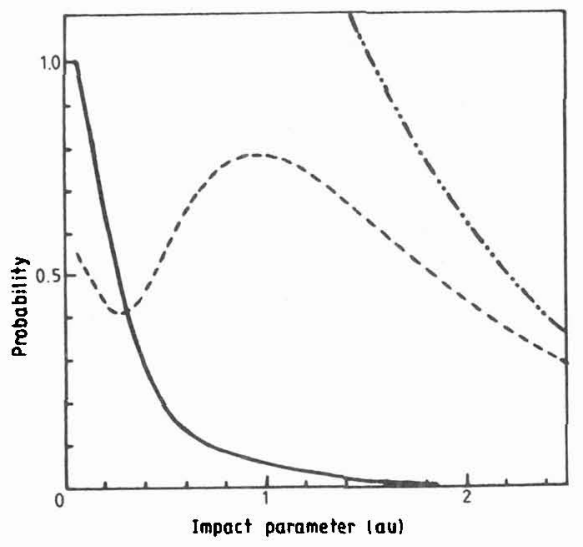

Fig. 4 - Probabilities of capture $P_{c}(\rho)$ and ionisation $P_{i}(\rho)$ of one electron of helium by $0^{8+}$ impact at $0.75 \mathrm{MEV} \mathrm{amu}^{-1},-\mathrm{P}_{\mathrm{c}}(\rho)$ normalised to 1 at $\rho=0.05$ au where its value is $0.298 ; \cdots, P_{i}(\rho)$ calculated by the MEDOC procedure (see text) with $\ell=0,1 ;-\cdots-P_{i}(\rho)$ calculated by the first Born approximation with $\ell=0, \ldots, 4$.

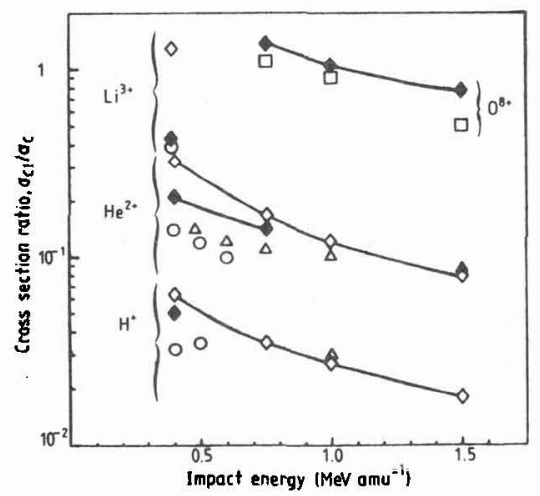

Fig. 5 - Ratio of the cross section of simultaneous capture and ionisation to the single capture cross section $\sigma_{c i} / \sigma_{c}$ as a function of the impact energy in $\mathrm{MeV} \mathrm{amu}^{-1}$ for various projectiles $\left(\mathrm{H}^{+}, \mathrm{He}^{2+}, \mathrm{Li}^{3+}, \mathrm{O}^{8+}\right)$. Theoretical predictions $/ 13 /$ : evaluation of capture by the CDW approximation and of ionisation by the Born approximation $(\diamond)$ and/or by the MEDOC treatment $(\bullet)$. Experimental data : $0, / 10 / ; \Delta, / 11 / ; \square, / 37 /$.

The agreement between our calculations and experiment is very good. Strong deviations from the Born approximation for $P_{i}$ can be observed. It is particularly striking in the case of $\mathrm{Li}^{3+}$ impact. Even for $\mathrm{H}^{+}$impact, we get a $25 \%$ difference at $400 \mathrm{keV}$, although for the same energy, the total ionisation cross section is in full agreement with the Born approximation $110 \%$. We note that our calculations with the MEDOC approach for $\mathrm{H}^{+}$and $\mathrm{He}^{+}$at $0.4 \mathrm{MeV}^{-1}$ show a discrepancy of $35 \%$ with the experimental data. Except in these last cases, our results show that the experimental data are compatible with an independent electron model if an acccurate evaluation is made within this model. Consequently, capture with ionisation can be used to probe the small impact parameter ionisation probability. 
Table 2 - Cross sections for single capture and for capture with ionisation of helium by ion impact (in $\left.\mathrm{cm}^{2}\right)^{+} \mathrm{a}^{\mathrm{b}}$ stands for $a \times 10^{\mathrm{b}}$

\begin{tabular}{|c|c|c|c|c|c|c|c|c|c|c|}
\hline & & & $\mathrm{W}+$ Born & & & N+MEDOC & & & xperimen & \\
\hline & Energy & & & & & & & & & \\
\hline Ion & $\left(\mathrm{MeV} a \mathrm{mu}^{-1}\right)$ & $\sigma_{c}$ & $\sigma_{c i}$ & $\sigma_{c i} / c_{c}$ & $\sigma_{c}$ & $\sigma_{c i}$ & $\sigma_{c i} / \sigma_{c}$ & $\sigma_{c}$ & $\sigma_{c i}$ & $\sigma_{c i} / \sigma_{c}$ \\
\hline $\mathrm{H}^{+}$ & 0.4 & $1.9^{-19}$ & $1.2^{-20}$ & $6.3^{-2}$ & $1.9^{-19}$ & $9.7^{-21}$ & $5.0^{-2}$ & $2.2^{-19}$ & $7.1^{-21}$ & $3.2^{-2}$ \\
\hline & 0.75 & $1.2^{-20}$ & $4.1^{-22}$ & $3.5^{-2}$ & - & - & - & - & - & - \\
\hline & 1.0 & $3.0^{-21}$ & $7.9^{-23}$ & $2.7^{-2}$ & - & - & - & - & .- & $3.0^{-2}$ \\
\hline & 1.5 & $4.0^{-22}$ & $7.3^{-24}$ & $1.8^{-2}$ & - & - & - & - & - & - \\
\hline $\mathrm{He}^{2+}$ & 0.4 & $2.1^{-18}$ & $7.1^{-19}$ & $3.3^{-1}$ & $2.3^{-18}$ & $4.9^{-19}$ & $2.1^{-1}$ & $3.1^{-18}$ & $4.3^{-19}$ & $1.4^{-1}$ \\
\hline & 0.75 & $1.8^{-19}$ & $2.9^{-20}$ & $1.64^{-1}$ & $1.8^{-19}$ & $2.6^{-20}$ & $1.42^{-1}$ & - & - & $1.1^{-1}$ \\
\hline & 1.0 & $5.0^{-20}$ & $6.1^{-21}$ & $1.21^{-1}$ & - & - & - & - & - & $1.0^{-1}$ \\
\hline & 1.5 & $7.7^{-21}$ & $6.1^{-22}$ & $7.9^{-2}$ & - & - & - & - & - & $8.5^{-2}$ \\
\hline $\mathrm{Li}^{3+}$ & 0.4 & $3.9^{-18}$ & $5.7^{-18}$ & 1.3 & $6.3^{\cdot 18}$ & $2.7^{-18}$ & $4.3^{-1}$ & $7.7^{-18}$ & $3.0^{-18}$ & $3.9^{-1}$ \\
\hline $0^{8+}$ & 0.75 & - & - & - & $5.1^{-18}$ & $6.9^{-18}$ & 1.37 & - & - & 1.1 \\
\hline & 1.0 & - & - & - & $1.8^{-13}$ & $1.9^{-18}$ & 1.05 & - & - & 0.9 \\
\hline & 1.5 & - & - & - & $3.7^{-19}$ & $2.8^{-19}$ & $7.7^{-1}$ & - & - & 0.5 \\
\hline
\end{tabular}

It is worth noting that in a recent work, Salin /16/ showed that similar conclusions can be drawn in studying single and double ionisation helium by multicharged ion impact at

$1.4 \mathrm{MeV} a \mathrm{am}^{-1}$. His framework is exactly the same as the one quoted above, i.e.: IEM2 and either the Born approximation or the MEDOC treatment with $\ell=0,1$ to get the ionisation probability. The single (S) and double (D) ionisation cross sections may be written as :

$$
\begin{aligned}
& \sigma_{s}=2 \pi \int_{0}^{\infty} \rho \mathrm{d} \rho 2 \mathrm{P}_{\mathrm{i}}(\rho)\left[1-\mathrm{P}_{\mathrm{i}}(\rho)\right] \\
& \sigma_{D}=2 \pi \int_{0}^{\infty} \rho \mathrm{d} \rho \mathrm{P}_{\mathrm{i}}^{2}(\rho)
\end{aligned}
$$

This work is particularly interesting since it is shown that saturation effects (due to non perturbative contributions) shows up even for single ionisation. Salin's results are quoted in table 3. The MEDOC procedure has two limitations

(i) Couplings with charge exchange channels are ignored

(ii) Salin's calculations are presently limited to $\ell=0,1$.

Therefore, one cannot expect MEDOC predictions to be reliable when capture processes are important. In the present application, CDWA calculations indicate that it might be the case for $z_{p} \geq 36 / 16 /$.

Table 3 - Ionization cross sections of helium by impact of ions with charge $Z_{p}$ at $1.4 \mathrm{Mev} / \mathrm{amu}\left(\right.$ in $\mathrm{cm}^{2}$ )

\section{Single ionization}

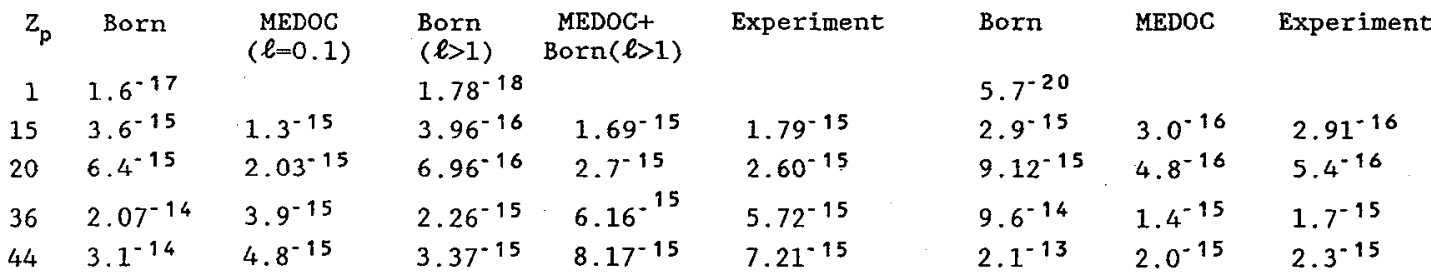


As to contributions of partial waves $\ell \geqslant 2$, they have been evaluated in the Born approximation for single ionisation. For $z_{p} z 36$, they appear to be comparable to MEDoC results for $\ell=0,1$ (see table 3). Although Salin's results are not fully reliable for $z_{p} Z 36$, they compare favourably to experimental data in any case. The difference between MEDOC and Born predictions for double ionisation is impressive, indicating that perturbative treatments are irrelevant to double (and even single) ionisation when the energy of the projectile is not high enough.

Thus, an independent electron model can be a prowerful tool to investigate non perturbative behaviours of the ionisation process in simultaneous capture and ionisation as well as in double ionisation by fast ion impact.

\section{5 - MULTIPLE IONISATION OF ATOMS BY FAST HIGHLY CHARGED ION IMPACTS}

Many experiments have been made in the last decade to investigate efficient ways to produce very slow beams of highly charged ions. The most promising technics to obtain ion beams with small spreads in energy and angle, seem to produce them by bombarding gas cells with fast beams of highly. charged ions $/ 18,40,41,42 /$.

In designing experiments in which secondary slow recoil ion beams are created by means of a primary beam of fast multicharged ions, one has to face many problems :

(i) The intensity of multicharged ion beams available on present-day high energy heavy ion accelerators.

(ii) The choice of targets intended for recoil ion production.

The ability to predict the characteristics of secondary recoil ion beams : intensity, mean kinetic energy, energy spread etc..., depends upon the energy, the charge and the stripping stage of projectiles. Kelbch et al /18/ produced highly stripped $\mathrm{Ne}^{+}$ions by impacts of $2 \mathrm{MeV}^{\mathrm{amu}}{ }^{-1} \mathrm{Brq}^{\prime+}$ ions in a neon gas cell. A sample of their results is displayed on fig. 6 . for $q^{\prime}=27$. They observed that multiple ionisation is due to pure direct multiple ionisation up to $q=5$. Since the $K$-shell capture processes rises above direct ejection of $k$-shell electrons, the highest stages of ionisation are reached through simultaneous ionisation of the L-shell and capture from the $\mathrm{K}$-shell. It is no wonder since it is well-known that the capture process is very efficient when the impact velocity $v$ is close to both the average electron velocity in the orbital where capture takes place and in the final orbital of capture $17 /$. It is easy to show that it is the case when the laboratory impact energy is :

$$
E\left(\mathrm{MeV} \mathrm{amu}{ }^{-1}\right) \simeq 0.05 \mid \epsilon_{i}(\text { a.u. })|\simeq 0.05| \epsilon_{f}\left(\text { a.u. }_{\text {. }}\right) \mid
$$

where $\epsilon_{i}$ and $\epsilon_{f}$ are respectively the initial and final orbital energies (such as Hartree-Fock ones) in atomic units.

$E$ is given in table 4 for the subshells of various elements. Values of $\epsilon_{j}$ axe from Clementi and Roetti's tables $/ 34 /$. Since $\mathrm{Br}^{27+}$ is an oxygen-like ion, it is easy to show that eq. (39)

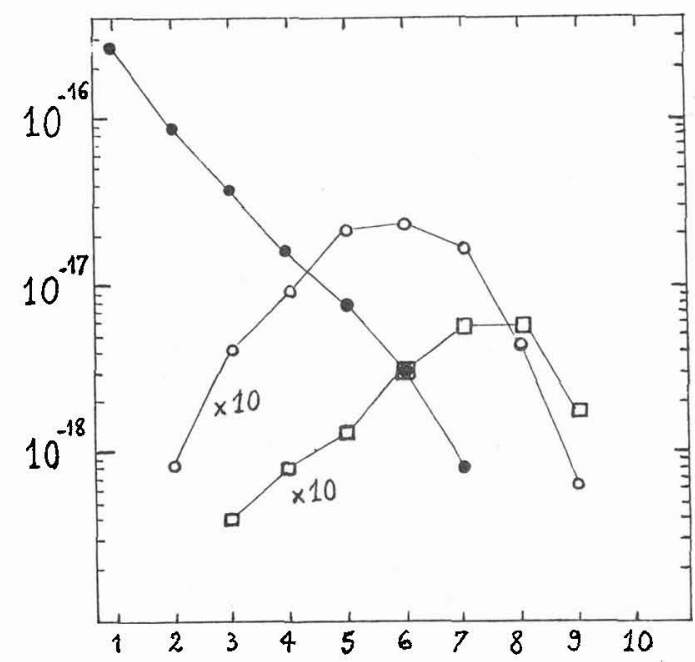

Fig. 6 - Cross sections $\sigma(q)$ for the production of $\mathrm{Ne}^{q+}$ recoil ions by $156 \mathrm{MeV} \mathrm{Br}^{27+}$ impact as a function of the recoil ion charge $q$. The final bromine charge state $q^{\prime}$ is : $q^{\prime}=27$ (ionisation processes only) ; $\mathrm{o}^{\prime}=26$ (one electron capture + ionisation); $\mathrm{q}^{\prime}=25$ (double electron capture + ionisation). For $q^{\prime}=25,26, \sigma(q)$ has been multiplied by $10 / 18 /$ 
is satisfied in the present case when the principal quantum number of the final orbital of $\mathrm{Br}^{26+}$ is $\mathrm{n}_{\mathrm{f}}=3$. Then, capture onto excited states of $\mathrm{Br}^{26+}$ is likely to be very effective.

Up to $q=6$, Kelbch et al used IEM2 to describe multiple ionisation. The ionisation probability $P_{L}(P)$ for the L-shell was taken to be the same for $s$ and $p$ orbitals:

$$
P_{L}(\rho)=P_{L}(0) \exp \left(\rho / r_{L}\right)
$$

Table 4 - Laboratory impact energy in $\mathrm{MeV}^{a m u^{-1}}$ for which the impact velocity is equal to the electron orbital one.

\begin{tabular}{|c|c|c|c|c|c|c|}
\hline & $\mathrm{He}$ & $\mathrm{Ne}$ & Ar & $\mathrm{kr}$ & $\mathrm{Xe}$ & $\mathrm{Br}^{26+}$ \\
\hline $1 \mathrm{~s}$ & 0.05 & 1.64 & 5.93 & 26.01 & 61.22 & 27.05 \\
\hline $\begin{array}{l}2 s \\
2 p\end{array}$ & & $\begin{array}{l}0.10 \\
0.04\end{array}$ & $\begin{array}{l}0.62 \\
0.48\end{array}$ & $\begin{array}{l}3.50 \\
3.15\end{array}$ & $\begin{array}{l}9.47 \\
8.89\end{array}$ & $\begin{array}{l}5.55 \\
5.28\end{array}$ \\
\hline $3 s$ & & & 0.06 & 0.54 & 2.01 & 7 \\
\hline $\begin{array}{l}3 p \\
3 d\end{array}$ & & & 0.03 & $\begin{array}{l}0.42 \\
0.19\end{array}$ & $\begin{array}{l}1.76 \\
1.31\end{array}$ & 2.03 \\
\hline $\begin{array}{l}4 s \\
4 p \\
4 d\end{array}$ & & & & $\begin{array}{l}0.06 \\
0.03\end{array}$ & $\begin{array}{l}0.39 \\
0.30 \\
0.14\end{array}$ & \\
\hline $\begin{array}{l}5 s \\
5 p\end{array}$ & & & & & $\begin{array}{l}0.05 \\
0.02\end{array}$ & \\
\hline
\end{tabular}

Then, the multiple ionisation cross section reads /6/

$$
\sigma_{q}=2 \pi \int_{0}^{\infty}\left(\begin{array}{l}
8 \\
q
\end{array}\right)\left[P_{L}(p)\right]^{q}\left[1-P_{L}(P)\right]^{8-q}
$$

The values $P_{L}(0)=0.75$ and $r_{L}=0.25$ a.u. were obtained by fitting the experimental data. From table 4, the energy appears very high for the $2 p$ subshell of neon. Then, the ionisation of the $2 p$ subshell is dominated by dipole terms in the interaction, i.e., by long range forces $/ 13,16 /$. Then, it is not surprising that formula (40), which fits the behaviour of $P_{L}(p)$ at large impact parameters, is able to nicely predict multiple ionisation for $q \leqslant 6$. In subsequent experiments $/ 40,41$, investigations were made toward the feasibility of recoil ions beams for further applications. Therefore, a need for knowledge of intensities, energy distributions and angular speads of recoil ions appeared $/ 40,42,43 /$. Until now, most theoretical predictions are made within uncorrelated electron models. Horbatsch and Dreizler $146 /$ investigated the ionisation of argon by $\mathrm{U}^{\prime+}\left(\mathrm{q}^{\prime}=65-75\right)$ at energies ranging from $3.6 \mathrm{MeV} \mathrm{amu}^{-1}$ to $1.5 .5 \mathrm{MeV} \mathrm{amu}^{-1}$. Their model is based on a time evolution of a many body system through quantum statistical methods. A qualitative agreement with experimental cross sections /41/ for the production of $\mathrm{Ar}^{\mathrm{q}^{+}}$ions as function of $\mathrm{q}$, is found. Although an IEM is not used by 01son et al $/ 45 /$, their description of multicharged ion-atom collisions replace the electron-electron interaction by effective charges in the interactions between the electrons and their parent nucleus. They used a n-body Classical Trajectory Monte Carlo Method ( $\mathrm{C}$ CTMCM) to look at $1.4 \mathrm{MeV}^{a^{-1}}$ collision of $\mathrm{U}^{32+}$ with neon. In addition to the above mentioned approximation, they assume that the projectile is considered as a point charge. For a n electron target, the model requires to numerically solve 6 (N +2 ) coupled first order differential equations. This heavy machinery provided for the first time angular distributions and energies of the recoil ion, the projectile ion and the ejected electrons. Mean recoil energies of $\mathrm{Ne}^{q+}$ ions as well as halfwidths of the energy spreads are in quite good agreement with experimental data. More important, Olson et al showed that recoil ions are produced at very low translational energies.

In the experiments quoted above, either the energy was not high enough to keep capture processes from intervening in the stripping process of the target $/ 18,42 /$ or the projectile charge was too high to use perturbative treatments (see section 4). However at the high impact energies of GANIL, multiple ionisation is expected to be due to ionisation processes

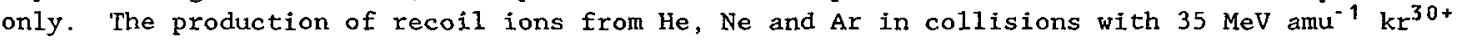
ions has been investigated recently /17/. Such a primary ion beam is normally available at GANIL. The beam velocity is deduced from the relativistic relation:

$$
v=c\left[1-\left(1+\frac{T}{931.48}\right)^{-2}\right]^{1 / 2}
$$


where $\mathrm{c}$ is the velocity of light, $\mathrm{T}$, is the laboratory impact energy in $\mathrm{MeV} a m u^{-1}$ and $931.48 \mathrm{MeV}$ is the average nucleon mass in nuclei. For $\mathrm{T}=35 \mathrm{MeV}$ amu ${ }^{-1}$, one has $v=36.40 \mathrm{a} . \mathrm{u}$. Then the investigations of Lesteven et al $/ 17 /$ have been made within IEM2. In order to show that high impact velocity conditions are fulfilled, we consider the worst situations that might be met. On the one hand, the highest target orbital velocity $v_{e, t}$ is found for the K-shell of argon. It is obtained easily from table 4 since the velocity of a $25 \mathrm{keV}$ proton is $1 \mathrm{a} . \mathrm{u}$. Thus one finds $v_{k} \simeq 15.4 \mathrm{a} . \mathrm{u}$. for argon. On the other hand the highest velocity on a bound orbital of $\mathrm{Kr}^{29+}$ is found to be $15.7 \mathrm{a} . \mathrm{u}$. on the $2 \mathrm{~s}$ subshel1 $134 /$. Thus one has :

$$
\frac{v_{e T}}{v} \leqslant 0.42 \quad ; \quad \frac{v_{e p}}{v} \leqslant 0.43
$$

The first ratio gives some confidence in the application of a Plane Wave Born Approximation (PWBA) for determining ionisation probabilities $P_{i}(\rho)$. However, for the ionisation of helium by $\mathrm{He}^{2+}$, Gayet and Salin /13/ found that PWBA give reliable predictions down to $0.75 \mathrm{MeV} \mathrm{amu}^{-1}$, i.e., for $\mathrm{v}_{\mathrm{e}} / \mathrm{v} \simeq 0.26$. Hence, direct ionisation of the $\mathrm{K}-\mathrm{shel1}$ of argon has not been considered in the work of Lesteven et al $/ 17 /$.

The eventual contribution of the capture process to the stripping of the target is easily discarded. Using CDWA, Lesteven et al /17/ show that the capture probability $P_{c}(\rho)$ in helium by $35 \mathrm{MeV}$ amu-1 $\mathrm{Kr}^{34+}$ ions is less than $10^{-4} P_{\mathfrak{i}}(\rho)$ everywhere. Using previous results $/ 7 /$, they show that the situation is the same with neon and argon targets. Thus, the production of multiply charged recoil ions is a result of direct ionisation processes. Then, the

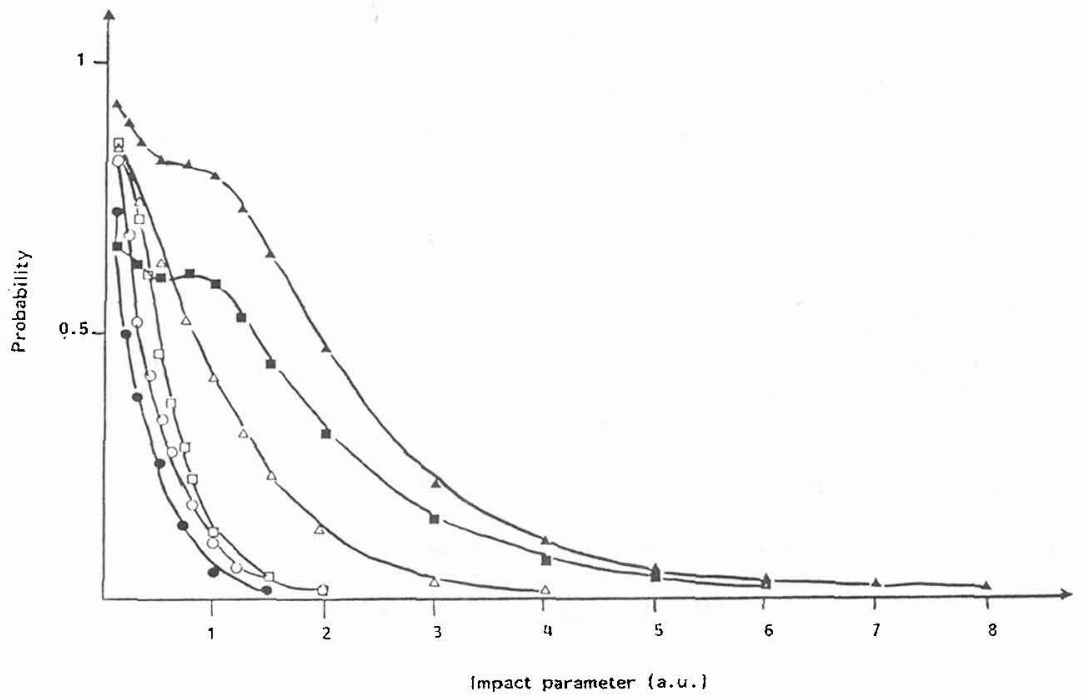

Fig. 7 - Probabilities of ejection of one electron $P_{n_{i}}, \ell_{i}\left|m_{i}\right|(P)$ of argon, by collision

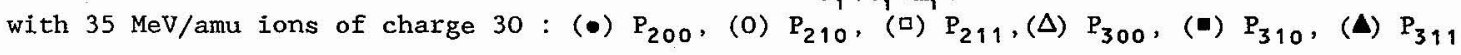

simultaneous ejection of several electrons from the target has been studied through IEM2/6/. The probability $P_{i}(P)$ is calculated with PWBA assuming that each ejected electron experiences the same potential from the rest of the target in both the initial and the final states. This assumption relies on the sudden change suffered by the target : except for the very fast ejected electrons, most of them are still in place while the projectile is far away. Moreover, fast ejected electron contributions to ionisation are very small. Hence, the interactions of electron in various subshells of the target have been represented by reliable model potentials $/ 44$ / which enable Lesteven et al to nicely reproduce the radial wave functions of Clementi and Roetti /34/. Therefore, calculations of the probabilities to eject one electron have been made for all subshells but the K-shell of argon. A sample of results is given by fig. 7 for the individual ejection probabilities in argon and by fig. 8 for the probabilities $P_{J}$ to remove a given number $J$ of electrons from argon. Multiple ionisation cross sections $\sigma_{J}$ have been predicted up to $J=16$ for argon. Some of them are quoted in table 5 . 


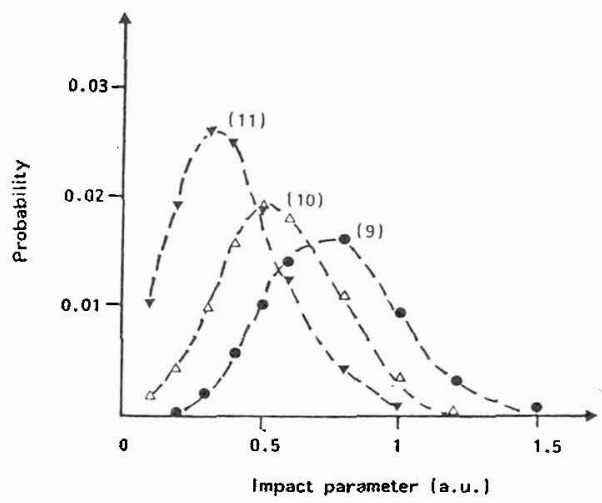

Fig. 8 - Probabilities to remove $\mathrm{J}$ electrons from argon : $\bullet=\mathrm{J}=9, \Delta \mathrm{J}=10, \boldsymbol{J}=11$ Symbols indicate the points where calculations have been made

Table 5 - Multiple ionisation cross sections for $35 \mathrm{MeV} \mathrm{am}^{-1} \mathrm{Kr}^{30+}$ impinging on various targets. a(b) stands for $a \times 10^{-b}$

$\begin{array}{rcccc}J & \text { target } & \text { He } & \text { Ne } & \text { Ar } \\ 2 & & 1.03(-16) & 2.06(-16) & 3.61(-16) \\ 10 & & & 8.96(-19) & 9.84(-19) \\ 16 & & & & 1.22(-19)\end{array}$

More interestingly, Lesteven et al predict the kinetic energy distribution of recoil ions directly from the knowledge of $P_{J}(\rho)$.

The demonstration is made in the laboratory frame by means of 4 reasonnable assumptions : 1 . The projectile trajectory is a quasi straight line

2 - The projectile is considered as a point charge $Z_{p}$ even though it could be a non-bare nucleus.

3 - The projectile-target interaction takes place through the Hartree-Fock potential of the neutral. target in its ground state.

4 - The displacement of the target during the collision is neglected.

The hypothesis 1 is known to be excellent. The hypothesis 4 is checked consistently at the end of calculations : it is excellent too. As to the assumption 2, it is good enough as long as the radius of the projectile ion is roughly smaller than the impact parameter. The radius of $\mathrm{Kr}^{30+}$ ions is about $0.18 \mathrm{a} . \mathrm{u}$. Then, one expects the results to be reliable for

$\rho \geq 0.2$ a.u. Finally the hypothesis 3 appears reasonnable since total ionisation cross sections are dominated by momenta of the ejected electrons much smaller than the impact velocity. Hence, the electronic density of charge around the target is almost unchanged at the end of the collision whatsoever the number of ejected electrons.

Now the force experienced by the target is opposed to the one sustained by the projectile, i.e. :

$$
\vec{F}_{T}=Z_{P} \underset{R}{\vec{\nabla}_{\vec{R}}} V_{H F}(R)=Z_{P} \frac{d V_{H F}(R)}{d R} \frac{\vec{R}}{R}
$$

where $V_{H F}(R)$ is a Hartree-Fock potential built in a standard way /47/ with Clementi and Roetti's wave functions $/ 34 / . \vec{R}$ is the internuclear distance given in (2). Then it is easy to show that the recoil momentum is :

$$
\overrightarrow{\mathrm{P}}_{\mathrm{T}}(\overrightarrow{\mathrm{\rho}})=2 \frac{\mathrm{Z}_{\mathrm{p}}}{\mathrm{V}} \vec{\rho} \int_{0}^{+\infty} \frac{\mathrm{dV}_{\mathrm{HF}}(\mathrm{R})}{\mathrm{dR}} \frac{\mathrm{dZ}}{\mathrm{R}}
$$


It is oriented along the $\vec{\rho}$ axis, i.e. perpendicular to the projectile trajectory. Further the distribution function, to produce ions of a given charge state $J$, as a function of $\rho$ is:

$$
D_{J}(\rho)=\frac{2 \pi}{\sigma_{J}} \rho P_{J}(\rho)
$$

All ions created for this impact parameterf have the same recoil energy

$$
\epsilon=\frac{P_{T}^{2}(\rho)}{2 M_{T}}
$$

Finally, it is easy to show that the energy distribution for a given charge state $J$ is :

$$
D_{J}(\epsilon)=-D_{J}(\rho)\left(\frac{d \epsilon}{d \rho}\right)^{-1}
$$

Thus, it is shown that, at high impact velocities

(i) target recoil occurs at $90^{\circ}$ to the direction of the incident bean.

(ii) the kinetic energy of recoil ions depends only upon the impact parameter $p$ (not upon their final charge $J$ ).

(iii) the energy distribution of recoil ions of charge $J$ is determined by the probability $P_{J}(\rho)$ of creating them.

The above conclusions last as long as the 4 above mentionned assumptions are valid. It is obvious that the angular distribution of recoil ions will be affected as soon as charge exchange contributions occur since the target-projectile interaction is not the same in the exit channel as in the entrance one.

Energy distributions for argon targets are displayed on figure 9. Due to the high velocity of the projectiles, one observes that the maximum of the distribution lies in the thermal noise at room temperature for $J \leqslant 10$. A general conclusion is that recoil energies are very small as already mentionned by olson et al $/ 42 /$. Thus, ion beams delivered at the GANI. facility provide us with ideal conditions to predict the characteristics of recoil ion beams by means of theoretical tools as simple as the PWBA and the IEM. It is worth noting that more sophisticated and much more costly methods must be employed to make similar predictions in different conditions $/ 43 /$.

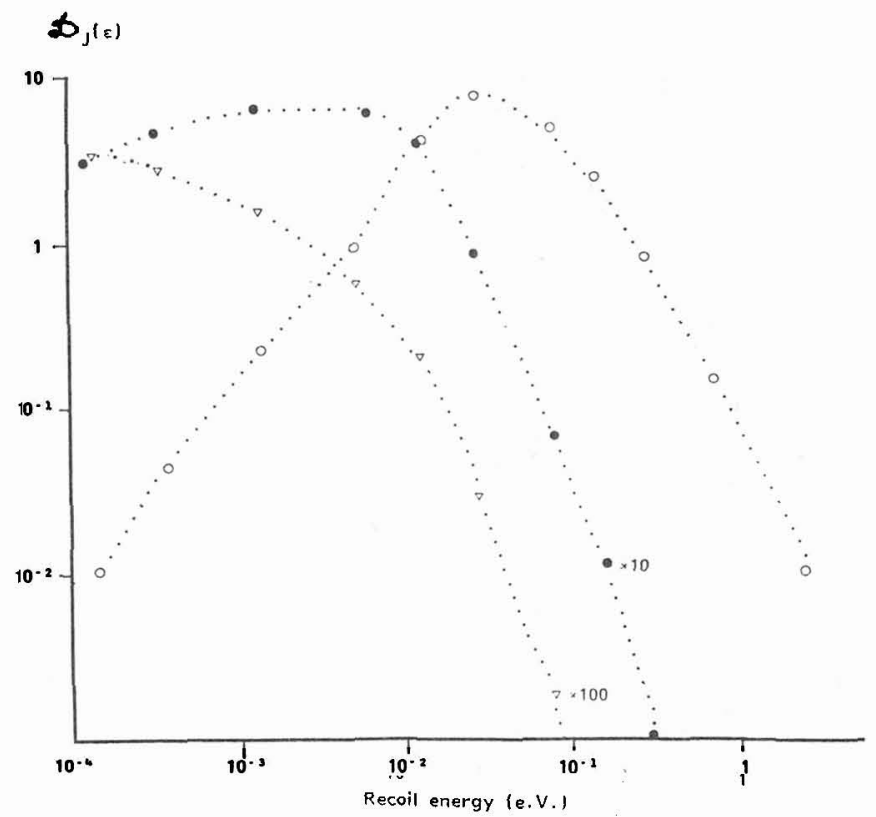

Fig. 9 - Distribution functions of recoil ions of charge $J, \$(\epsilon)$ obtained by collision of $35 \mathrm{MeV} / \mathrm{amu}$ ions of charge $\mathrm{Z}_{\mathrm{p}}=30$ : with argon. $(\nabla) \mathrm{J}=6,(\bullet) \mathrm{J}=8,(0) \mathrm{J}=11$ 
6 - CONCLUSION

We have shown that the Independent Electron Model is a valuable tool to investigate multiple electron processes at high impact velocities when the charges of the nuclei are high enough to ignore electron-electron correlation effects.

\section{REFERIENCES}

/1/ R. Gayet, Nuc1. Science Applications $\underline{1}$ (1983) 555

/2/ J.R. Macdonald, S.M. Ferguson, T. Chiao, L.D. Ellsworth and S.A. Savoy, Phys. Rev. A $\underline{5}$ (1972) 1188

/3/ H.D. Betz, Rev. Mod. Phys. 44 (1972) 465

/4/ S.M. Ferguson, J.R. Macdonald, T. Chiao, L.D. E1lsworth and S.A. Savoy, Phys. Rev. A $\underline{8}$ (1973) 2417

15/ F.W. Martin and J.R. Macdonald, Phys. Rev. A4 (1971) 1974

/6/ J.H. McGuire and O.L. Weaver, Phys. Rev. A16 (1977) 41

17/ Dz. Belkić, R. Gayet and A. Salin, Phys. Rep. 56 (1979) 279

/8/ K. Wohrer, F. Ben Salah, J.P. Rozet, A. Chetioui, A. Touati, P. Bouisset, D. Vernhet, C. Stephan and R. Gayet, Nucl. Instrum. and Meth. B27 (1987) 594

/9/ R. Gayet, R.D. Rivarola and A. Salin, J. Phys. B 14 (1981) 2421

/10/ M.B. Shah and H.B. Gilbody, J. Phys. B 18. (1985) 899

/11/ H. Knudsen, L.H. Andersen, P. Hvelplund, J. Sprensen and D. Ćirić, J. Phys. B 20 (1987) L253

/12/ J.A. Tanis, M.W. Clark, R. Price, S.M. Ferguson and R.E. Olson, Nucl. Instrum. Meth. B 23 (1987) 167

113/ R. Gayet and A. Salin,. J. Phys. B 20 (1987) L571

/14/ B. Brendlé, R. Gayet, J.P. Rozet and K. Wohrer, Phys. Rev. Letters 54 (1985) 2007

115/ K. Wohrer, A. Chetioui, J.P. Rozet, A. Jolly, F. Fernandez, C. Stephan, B. Brendlé and R. Gayet, J. Phys. B $\underline{19}$ (1986) 1997

/16/ A. Salin, Phys. Rev. A 36 (1987) 5471

17/ I. Lesteven-Vaïsse, D. Hennecart and R. Gayet, J. Phys. France (1988) in press

18/ S, Kelbch, H. Schmidt-Böcking, J, Ulirich, R. Schuch, E. Justiniano, H. Ingwersen and C.L. Cocke, Z. Phys. A 317 (1984) 9

19/ I. Ben-Itzhak, T.J. Gray, J.C. Legg and J.H. McGuire, Phys. Rev. A37 (1988) 3685

$120 /$ C.D. Lin, Phys. Rev. A 19 (1979) 1510

121/ J.R. Oppenheimer, Phys. Rev, 31 (1928) 349

122/ H.C. Brinkman and H.A. Kramers, Proc. Ac. Sci. Amsterdam 33 (1930) 973

/23/ C.W. Woods, R.L. Kauffman, K.A. Jamison, N. Stolterfoht and P. Richard, Phys. Rev. A 12 (1975) 1393

/24/ K. Roy, S.C. Mukherjee and D.P. Sural, Phys. Rev, A 13 (1976) 987

/25/ J.F. Williams, Phys. Rev, 157 (1967) 97

/26/ L.H. Toburen and M.Y. Nakai, Phys. Rev. 177 (1969) 191

127/ V.I. Gerasimendko, Sov. Phys. JETP 14 (1962) 789

/28/ DL. Belkik, R. Gayet, J. Hanssen and A. Salin, J. Phys. B 19 (1986) 2945-53

/29/ R. Gayet J. Phys. B $\underline{5}$ (1972) 483

$/ 30 /$ R. Gayet, Thèse de Doctorat d'Etat, Bordeaux (1973) unpublished

/31/ I.M. Cheshire, Proc. Phys. Soc. 84 (1964) 89

/32/ Dž. Belkić and R.K. Janev, J. Phys. B $\underline{6}$ (1973) 1020

/33/ DŽ. Belkic, R. Gayet and A. Salin, Comput. Phys. Commun. 23 (1981) 153 ; Comput. Phys. Commun. 30 (1983) 193 ; Comput. Phys. Commun. 32 (1984) 385

/34/ E. Clementi and C. Roetti, At. Data Nucl. Data Tables, 14 (1974) 177

135/ T.C. Theisen and J.H. McGuire, Phys. Rev. A20 (1979) 1406

/36/ E.W. McDaniel, M.R. Flannery, H.W. Ellis, F.L. Eisele and W. Pope US Army Missile Research and Development Command Technical Report H 78-1 (1977) L253

/37/ J.A. Tanis, M.W. Clark, R. Price, S.M. Feguson and R.E. Olson, Nucl. Instrum. Meth. B 23 (1987) 167

/38/ R. McCarroll and A. Salin, J. Phys. B 1 (1968) 163-71; J. Phys. B 11 (1978) L693

139/ J.M. Cheshire and E.C. Sullivan, Phys. Rev. 160 (1967) 4

/40/ J. Ullrich, C.L. Cocke, S. Kelbch, R. Mann, P. Richard and H. Schmidt-Böcking, J. Phys. B 17 (1984) L785

/41/ S. Kelbch, J. Ullrich, R. Mann, P. Richard and H. Schmidt-Böcking, J. Phys. B 18 (1985) 323

/42/ R.E. O1son, J. Ul1rich and H. Schmidt-Böcking, J. Phys. B 20 (1987) L809

/43/ M. Horbatsch and R.M. Dreizler, Phys. Letters 113A (1985) 251

/44/ M. Klapisch, Comput. Phys. Comm. 2 (1971) 239 ; C. Bottcher, J. Phys. B 6 (1973) 2368 